\title{
A New Hybrid Active Power Filter Topology Enabling Low Voltage Switching Devices and Storage Capacitors
}

\author{
Marques H.S. ${ }^{1}$, Anunciada A.V. ${ }^{2}$ \\ Instituto de Telecomunicações - Instituto Superior Técnico \\ Av. Rovisco Pais, 1 1049-001 LISBOA, PORTUGAL \\ Phone: +351 2184184 54, Fax: +351 2184184 72, e-mail ${ }^{1}$ : hdsm@lx.it.pt; e-mail²: avaa@lx.it.pt
}

\begin{abstract}
The increasing importance of mains harmonic content has been responsible for several improvements in Active Power Filter topologies in the last decade.

The pure multiple half-bridge configurations are now becoming replaced by new hybrid topologies, aiming to be simultaneously less bulky and expensive.

This new technique consists in reducing the voltages at the storage capacitors and consequently at the switching devices. This is achieved by introducing an extra filter between mains and the power electronics converter, tuned at the interest frequencies.

This paper presents a new topology for parallel hybrid active filters that can reduce the converter voltage down to $5 \%$ of the mains voltage magnitude.

A simple, comparative study will be presented, taking in consideration some topologies previously proposed by other authors.
\end{abstract}

\section{Keywords}

Active power filters, Power quality, Power electronics.

\section{Introduction}

The huge boom observed in the use of electronic devices, in the last decades, has astonishing increased the mains harmonic content.

The consequent undesirable effects are the primary cause of:

- electromagnetic compatibility problems,

- sine wave distortion,

- extra dissipative losses,

- excessive neutral currents, even in equilibrated three-phase loads.

Therefore a great amount of effort has been made in order to minimize all those unwanted and quite expensive effects. intend:

Two different policies have been taken to reach this

- regulations impose limitations to the input current waveform of electronic products, according to their use, maximum input power and specific characteristics and purposes.

- development of electronic systems specifically intended to compensate the mains harmonic content by means of specific current injection.

On the second case, once the main purpose is to cancel high order harmonics, the currents injected should have a frequency spectrum equal in amplitude and in opposite phase to those of the currents present in the mains network. This is true for all frequencies, except for the fundamental, that should not be compensated.

This last principle can be accomplished by different topologies resulting in more or less expensive, and more or less bulky solutions that must be considered according to their specific applications.

The objective of this paper is to present a new topology of an active hybrid shunt active filter and to proceed to a discussion and comparison with solutions previously proposed by other authors, [1].

In order to clarify the presentation, a previous study about general active power filter topologies is also made to provide appropriated tools for further analysis.

Study results show that in this new topology it is possible to have a $95.8 \%$ converter voltage reduction, when compared to the corresponding pure active solutions.

A first consequence is the significant reduction of filter size and cost.

Another advantage is to make the use of low voltage rated converters, possible even in medium voltage applications.

\section{The Pure Shunt Active Power Filter}

The circuit of Fig. 1 represents the basic configuration of a pure active filter. 
Considering that mains voltage $\boldsymbol{v}_{\boldsymbol{m}}$ is purely sinusoidal:

$$
v_{m}=V_{m} \sin \left(\omega_{1} t\right)
$$

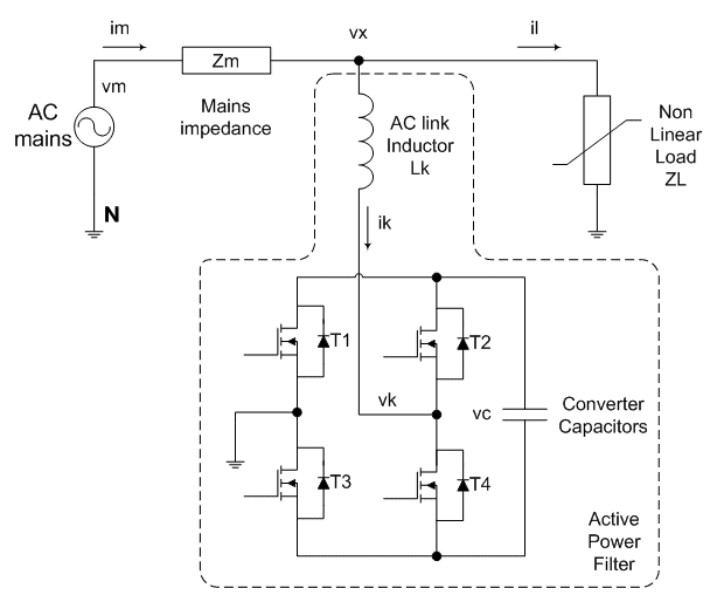

Fig. 1 - A system configuration of a pure shunt active filter

The non-linear load current $\boldsymbol{i}_{\boldsymbol{l}}$ can be written as a Fourier series:

$$
\begin{aligned}
& i_{l}=I_{1} \cdot \sin \left(\omega_{1} t+\phi_{1}\right)+\sum_{k=2}^{\infty} I_{k} \cdot \sin \left(k \omega_{1} t+\phi_{k}\right) \\
& i_{l}=\underbrace{I_{10} \cdot \sin \left(\omega_{1} t\right)}_{\begin{array}{c}
\text { active power } \\
\text { component }
\end{array}}+\underbrace{I_{11} \cdot \cos \left(\omega_{1} t\right)+\sum_{k=2}^{\infty} I_{k} \cdot \sin \left(\omega_{k} t+\phi_{k}\right)}_{\begin{array}{c}
\text { reactive power and } \\
\text { distortion }
\end{array}} \\
& i_{l}=i_{a}+i_{d}
\end{aligned}
$$

where $\boldsymbol{i}_{\boldsymbol{a}}$ is the active component of the first harmonic and $\boldsymbol{i}_{\boldsymbol{d}}$ is the sum of the reactive component and higher harmonics.

The converter control method will be such that validates the following expression:

$$
i_{k}=-i_{d}
$$

Making this assumption it is easy to verify that the mains current will result in its active component.

$$
\begin{aligned}
& i_{m}=i_{l}+i_{k}=i_{a}+i_{d}+\left(-i_{d}\right)=i_{a} \\
& i_{m}=I_{10} \cdot \sin \left(\omega_{1} t\right)
\end{aligned}
$$

This means that the non-linear load plus the active power filter combination actuates as a pure resistive linear load.

Neglecting mains impedance $\boldsymbol{Z}_{\boldsymbol{m}}$, and assuming that $\boldsymbol{v}_{\boldsymbol{x}}$ is approximately equal to $\boldsymbol{v}_{\boldsymbol{m}}$ : the ideally null average power requested by the active filter can be evaluated.

$$
P_{k}=\frac{\omega}{2 \pi} \int_{0}^{2 \pi} v_{m}(\omega t) \cdot i_{k}(\omega t) d \omega t
$$

$$
\begin{aligned}
P_{k}= & \frac{\omega}{2 \pi} \int_{0}^{2 \pi} V_{m} \cdot \sin \left(\omega_{1} t\right) \cdot\left[I_{11} \cdot \cos \left(\omega_{1} t\right)+\sum_{k=2}^{\infty} I_{k} \cdot \sin \left(\omega_{k} t+\phi_{k}\right)\right] d \omega t \\
P_{k}= & \frac{\omega}{2 \pi}\{V_{m} \cdot I_{11} \underbrace{\int_{0}^{2 \pi} \sin \left(\omega_{1} t\right) \cdot \cos \left(\omega_{1} t\right) d \omega t}_{=0}+ \\
& \left.\sum_{k=2}^{\infty}[I_{k} \cdot V_{m} \cdot \underbrace{\int_{0}^{2 \pi} \sin \left(\omega_{1} t\right) \cdot \sin \left(k \omega_{1} t+\phi_{k}\right) d \omega t}_{=0}]\right\} \\
P_{k}=0 & \Rightarrow P_{m}=P_{l}
\end{aligned}
$$

The converter operation that satisfies (2.3), is calculated by the equation:

$$
v_{k}=v_{x}-L_{k} \frac{d i_{k}}{d t}
$$

According to (2.6) and assuming, once again, that $v_{x} \approx v_{m}$, is:

$v_{k}=V_{m} \sin \left(\omega_{1} t\right)-L_{k}\left(I_{11} \varpi_{1} \sin \left(\omega_{1}\right)+\sum_{k=2}^{\infty} I_{k} k \omega_{1} \cos \left(k \omega_{1}+\phi_{k}\right)\right)$

Considering the trivial case $\boldsymbol{I}_{\boldsymbol{d}}=0$, it is rapidly denotable that: in order to satisfy (2.7), $\boldsymbol{v}_{\boldsymbol{k}}$ should assume values at least of the same magnitude of $\boldsymbol{V}_{\boldsymbol{m}}$. This implies that both switching devices and capacitors must be rated for voltage magnitudes higher then mains voltage peak amplitude. When the major objective is to minimize the dimensions and, consequently, the converter costs, this is actually an important limitation.

Inspecting the converter topology, (Fig. 1) and considering all interesting switching possibilities, it follows:

$$
v_{k}= \begin{cases}+V_{C} & \begin{cases}T_{2}, T_{3} & \text { on } \\
T_{1}, T_{4} & \text { off }\end{cases} \\
0 & \left\{\begin{array} { l l } 
{ T _ { 1 } , T _ { 2 } } & { \text { on } } \\
{ T _ { 3 } , T _ { 4 } } & { \text { off } }
\end{array} \text { or } \left\{\begin{array}{ll}
T_{3}, T_{4} & \text { on } \\
T_{1}, T_{2} & \text { off }
\end{array}\right.\right. \\
-V_{C} & \begin{cases}T_{1}, T_{4} & \text { on } \\
T_{2}, T_{3} & \text { off }\end{cases} \end{cases}
$$

Actually the converter cannot exactly satisfy (2.7), since $\boldsymbol{v}_{\boldsymbol{k}}$ will only assume three different discrete values.

However, considering (2.6) the current $\boldsymbol{i}_{\boldsymbol{k}}$ can be rewritten as:

$$
\begin{aligned}
& \frac{d i_{k}}{d t}=\frac{v_{x}-v_{k}}{L_{k}} \\
& i_{k}=i_{k 0}+\frac{1}{L_{k}} \int_{t_{0}}^{t} v_{x}-v_{k} d t
\end{aligned}
$$

It becomes that if $\boldsymbol{v}_{\boldsymbol{k}}$ is modulated at a slightly higher frequency than the time constant $1 / \boldsymbol{L}_{\boldsymbol{k}}$, and averagely satisfies (2.7), the current $\boldsymbol{i}_{\boldsymbol{k}}$ verifies (2.3), except for a certain ripple current amplitude that can be 
made as small as desired. This ripple amplitude is directly related to the switching frequency, inductance $\boldsymbol{L}_{\boldsymbol{k}}$ and the DC capacitor voltage $\boldsymbol{V}_{\boldsymbol{c}}$.

\section{The Hybrid Shunt Active Power Filter}

A basic system configuration of an hybrid shunt active filter is presented in Fig. 2.

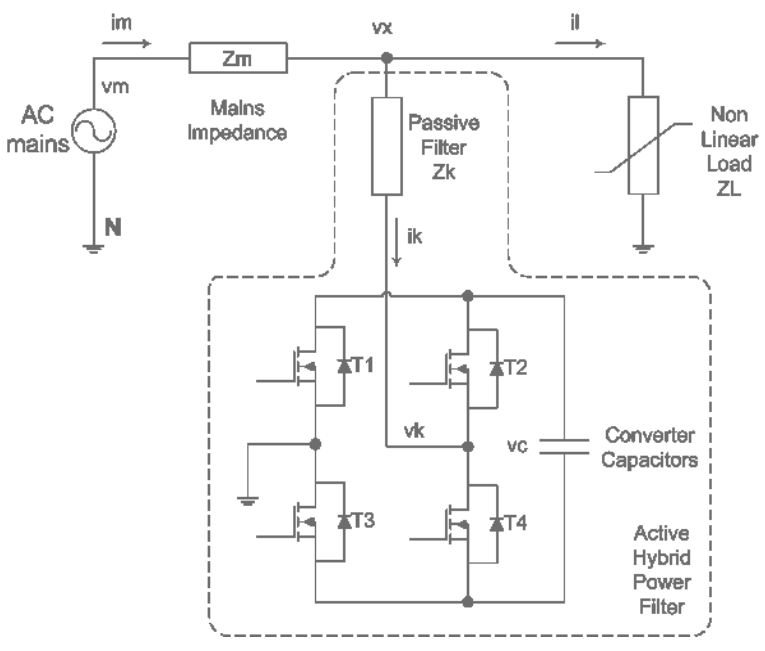

Fig. 2 - A system configuration of a hybrid shunt active filter

As in the previous chapter, equations (2.1) and (2.2) are still valid.

$$
\begin{gathered}
v_{m}=V_{m} \sin \left(\omega_{1} t\right) \\
i_{l}=I_{1} \cdot \sin \left(\omega_{1} t+\phi_{1}\right)+\sum_{k=2}^{\infty} I_{k} \cdot \sin \left(k \omega_{1} t+\phi_{k}\right) \\
=\underbrace{I_{10} \cdot \sin \left(\omega_{1} t\right)}_{\text {active power }}+\underbrace{I_{11} \cdot \cos \left(\omega_{1} t\right)}_{\text {reactive power }}+\underbrace{\sum_{k=2}^{\infty} I_{k} \cdot \sin \left(\omega_{k} t+\phi_{k}\right)}_{\text {harmonic distortion }} \\
=i_{10}+i_{11}+i_{h}
\end{gathered}
$$

It will be now considered that current component $\boldsymbol{i}_{\boldsymbol{h}}$ will be the only one to be compensated. The immediate consequence of this assumption is that, although mains current become approximately sinusoidal, the power factor will not be unitary except if the fundamental harmonic of the load current is in phase with voltage.

This is not as limitative as it may appear, because several applications, full-wave rectifiers for example, fulfill this condition. On the other hand, in the absence of harmonic content, power factor can easily be compensated with a capacitor bank. become:

$$
\begin{aligned}
& i_{l}=i_{10}+i_{11}+i_{h}, \\
& i_{k}=-i_{h}, \\
& i_{m}=i_{10}+i_{11}
\end{aligned}
$$

The active filter will no longer have to process energy at the fundamental frequency. At a first sight this may not appear to represent a significant advantage. However, the opposite conclusion occurs when the Laplace transform is computed for all frequencies:

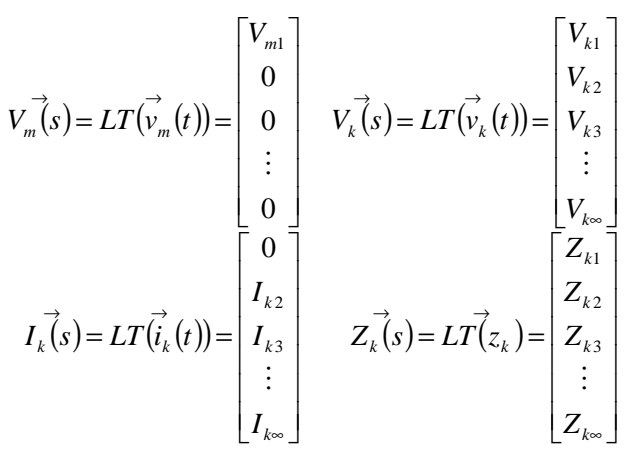

where the vector elements are complex, representing both amplitude and phase frequency components.

Computing $\vec{V}_{k}$ yields:

$$
\vec{V}_{k}=\vec{V}_{m}-\vec{Z}_{k} \vec{I}_{k} \Leftrightarrow\left[\begin{array}{c}
V_{k 1} \\
V_{k 2} \\
V_{k 3} \\
\vdots \\
V_{k \infty}
\end{array}\right]=\left[\begin{array}{c}
V_{m 1} \\
0 \\
0 \\
\vdots \\
0
\end{array}\right]-\left[\begin{array}{c}
Z_{k 1} \\
Z_{k 2} \\
Z_{k 3} \\
\vdots \\
Z_{k \infty}
\end{array}\right]\left[\begin{array}{c}
0 \\
I_{k 2} \\
I_{k 3} \\
\vdots \\
I_{k \infty}
\end{array}\right]
$$

Exploring the singularity of the equation above, it is possible to make $\boldsymbol{V}_{\boldsymbol{k} \mathbf{1}} \cong 0$, when $\left|\boldsymbol{Z}_{\mathbf{k} \mathbf{1}}\right|$ tends to infinite. This is only possible because it is assumed that no energy is processed at fundamental frequency.

The elimination of the term $\boldsymbol{V}_{\boldsymbol{k} \boldsymbol{1}}$ will radically reduce the converter voltage magnitude, making possible the use of lower voltage rated switching devices and capacitors.

The Laplace transform voltage vector, $\vec{V}_{k}$ reaches it's lowest possible minimum value when the frequency behavior of $\vec{Z}_{k}$ is given by the curve represented in the Fig. 3.

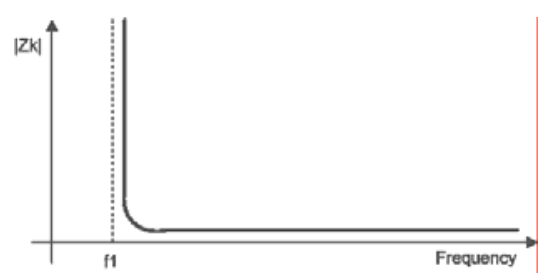

Fig. 3 -Ideal passive filter response 
In practice this is equivalent to replace the ac link inductor of Fig. 1 by a more complex passive association with the high pass characteristic shown above.

\section{Some Considerations About an Hybrid Shunt Active Filter Proposed in [1]}

The circuit present in Fig. 4 is a hybrid tree-phase active filter, proposed in [1]:

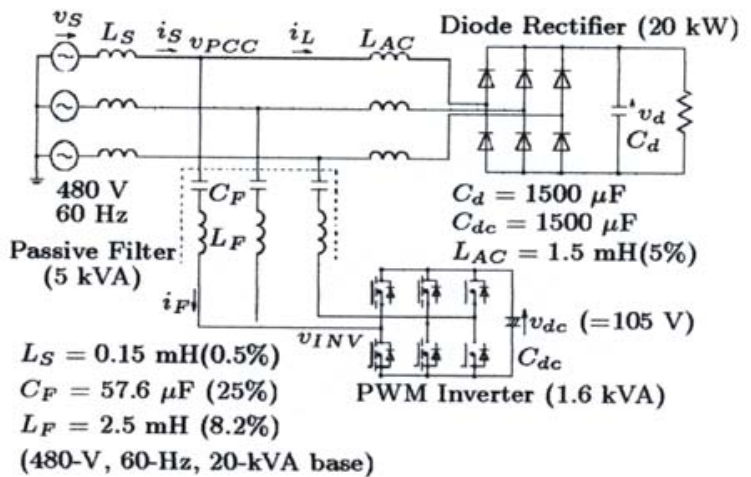

Fig. 4 - The configuration of a hybrid shunt active filter

The pairs $\boldsymbol{L}_{f}, \boldsymbol{C}_{\boldsymbol{f}}$ are easily identified as the passive filter $\boldsymbol{Z}_{\boldsymbol{k}}$, discussed in the previous chapter.

The response shown in Fig. 5 is, in fact, a good approximation to the ideal response (represented by a tiny stripe), permitting to reduce the required maximum peak voltage in the converter output from $480 \mathrm{~V}$ to $105 \mathrm{~V}$.

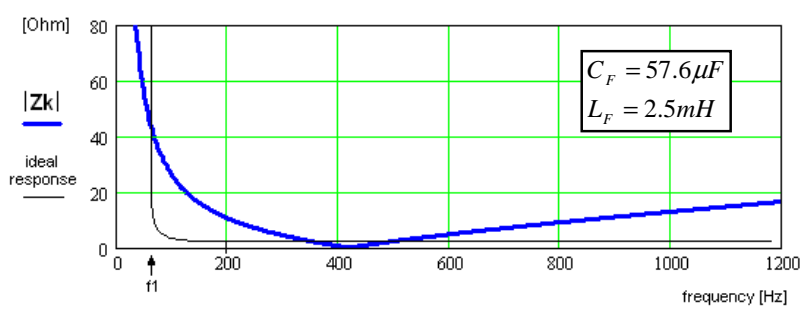

Fig. 5 - Proposed passive filter response Hirofumi Akagi - IEEE / 2003

Ideally, according to equation (3.5), a filter with an ideal response as shown above, would allow to operate the converter at almost null voltage. This means that the lower the real filter and ideal response curves difference is, the lower is the voltage at which the converter must operate.

However, some considerations must be made:

- As desired, $\left|Z_{k}\right|$ is low at high order frequencies but is not null, except for the resonant frequency near $420 \mathrm{~Hz}$.
- At the fundamental harmonic - $60 \mathrm{~Hz}$ in this example- $\left|Z_{k}\right|$ assumes high but finite values.

- The parameters $\boldsymbol{L}_{\boldsymbol{f}}$ and $\boldsymbol{C}_{\boldsymbol{f}}$ can be adjusted in order to set the resonant frequency (lowest $\left|\boldsymbol{Z}_{\boldsymbol{k}}\right|$ ) at the most significant harmonic, minimizing the vector product (3.5). This is only possible in applications where the loads are invariant and well determined.

- To achieve full power factor correction an extra reactive passive shunt element will be necessary if the load current component $\boldsymbol{i}_{\mathbf{1 1}}$ is not null.

- Fixing an appropriated resonant frequency: fitting the parameters $\boldsymbol{L}_{\boldsymbol{f}}$ and $\boldsymbol{C}_{\boldsymbol{f}}$ in order to increase $\left|\boldsymbol{Z}_{\boldsymbol{k}}\right|$ at the fundamental harmonic, necessarily also conducts to an unwanted increase of $\left|Z_{k}\right|$ for all other harmonics. On the other hand $\left|\boldsymbol{Z}_{\boldsymbol{k}}\right|$ also becomes decreased each time $\boldsymbol{L}_{f}$ and $\boldsymbol{C}_{\boldsymbol{f}}$ are fitted to reduce the impedance at the frequencies other than the fundamental.

The last point is of most importance since, no matter how many different $\boldsymbol{L}_{f}, \boldsymbol{C}_{f}$ combinations are tried, there will always exist a specific converter voltage value under which the converter will not operate properly.

Although the results presented in [1] are excellent, this limitation is very severe for example in singlephase applications, or unbalanced three-phase systems where the third harmonic, in contrast to Fig. 4, has a significant magnitude. In those cases, the converter voltage reduction will outcome much less suitable, considering that is exactly at low order harmonics, $\left(2^{\text {nd }}\right.$ and $3^{\text {rd }}$, non-existent in balanced three-phase systems), where the difference to the ideal response is more critical.

Next chapter proposes a new filter topology specially designed to overcome the limitations previously discussed.

\section{A New Filter Topology For Use In Hybrid Shunt Active Power Filters}

The new proposed filter topology is shown in Fig. 6. The major difference consists in an extra inductor $\boldsymbol{L}_{\boldsymbol{k} \mathbf{1}}$, in the passive filter association.

Filter response, considering $\boldsymbol{C}_{\boldsymbol{k}}$ with the same value as proposed in [4], is presented in Fig. 7.

The first advantage is that $\left|Z_{\boldsymbol{k}}\right|$ is no longer limited as in the previous solution. As in the ideal response, $\left|Z_{k}\right|$ actually tends to infinite at the fundamental frequency.

The second and probably the major advantage is that: it is now possible to decrease $\left|Z_{k}\right|$ for all frequencies above the fundamental without loosing the equality $\left|Z_{k}\right|\left(f=f_{1}\right)=\infty$. 


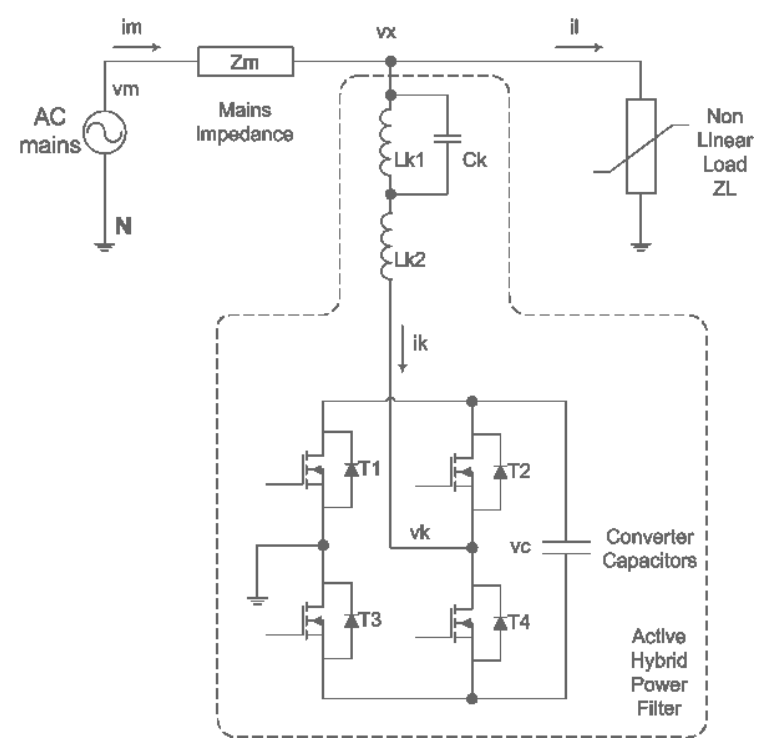

Fig. 6 - New Filter Topology

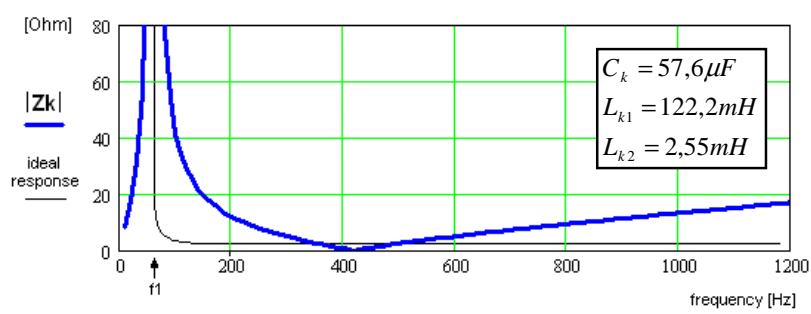

Fig. 7 -Frequency response of the new filter

In other words the new filter topology response can be settled to infinite at the fundamental frequency and made as low as desired for all other harmonics when the appropriated set of parameters $\boldsymbol{L}_{\boldsymbol{f} 1}, \boldsymbol{L}_{\boldsymbol{f} 2}$ and $\boldsymbol{C}_{\boldsymbol{f}}$ is taken.

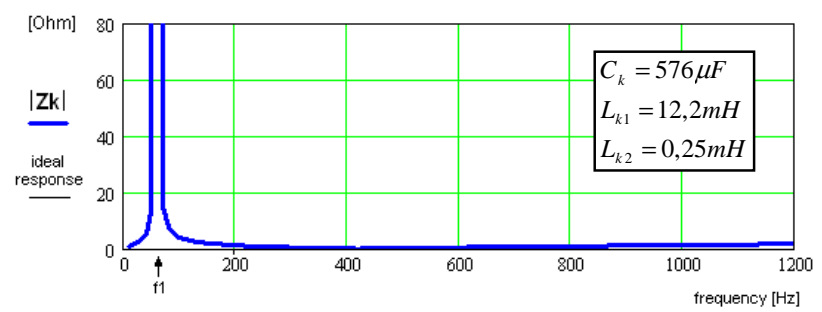

Fig. 8 - Frequency response of the new filter with a different set of parameters

In practice the filter parameters will be physically limited for the reason that the better the filter performance the larger will be the passive components.

Taking into account the frequency $\boldsymbol{\omega}_{\boldsymbol{h} \_ \text {max }}$ of the most significant harmonic and mains fundamental angular frequency $\omega_{1}$, it is possible to set the passive filter parameters relationship.

The passive filter impedance $\boldsymbol{Z}_{\boldsymbol{k}}$ of Fig. 6 , is given by expression (5.1):

$$
Z_{k}(j \omega)=\frac{j \omega\left(L_{k 1}+L_{k 2}-L_{k 1} L_{k 2} C_{k} \omega^{2}\right)}{1-L_{k 1} C_{k} \omega^{2}}
$$

A first zero occurs at $\omega_{z 1}=0 \mathrm{rad} \cdot \mathrm{s}^{-1}$. The pole is located at $\omega_{p}=\frac{1}{\sqrt{L_{k 1} C_{k}}}$. And the second zero at $\omega_{z 2}=\sqrt{\frac{L_{k 1}+L_{k 2}}{L_{k 1} L_{k 2} C_{k}}}$.

At the angular frequency $\omega_{p}$, the filter impedance will tend to infinite. For this reason $\omega_{p}$ is settled to be equal to the fundamental frequency, $\omega_{p}=\omega_{1}$, resulting:

$$
L_{k 1}=\frac{1}{C_{k} \omega_{1}^{2}}
$$

The frequency of the second zero is chosen in order to minimize (3.5). A simple and efficient way to accomplish it, is to make $\omega_{\mathbf{z} 2}=\omega_{h_{-} \text {max }}$. This yields to:

$$
L_{k 2}=\frac{L_{k 1}}{L_{k 1} C_{k} \omega_{h_{-} \max }^{2}-1}=\frac{1}{C_{k}\left(\omega_{1}^{2} \omega_{h_{-} \max }^{2}-1\right)}
$$

The initially assumed value of capacitance $\boldsymbol{C}_{\boldsymbol{k}}$, can satisfy an additional condition: $\left|Z_{k}\left(\omega=\omega_{x}\right)\right|<\zeta_{a x}$, for example.

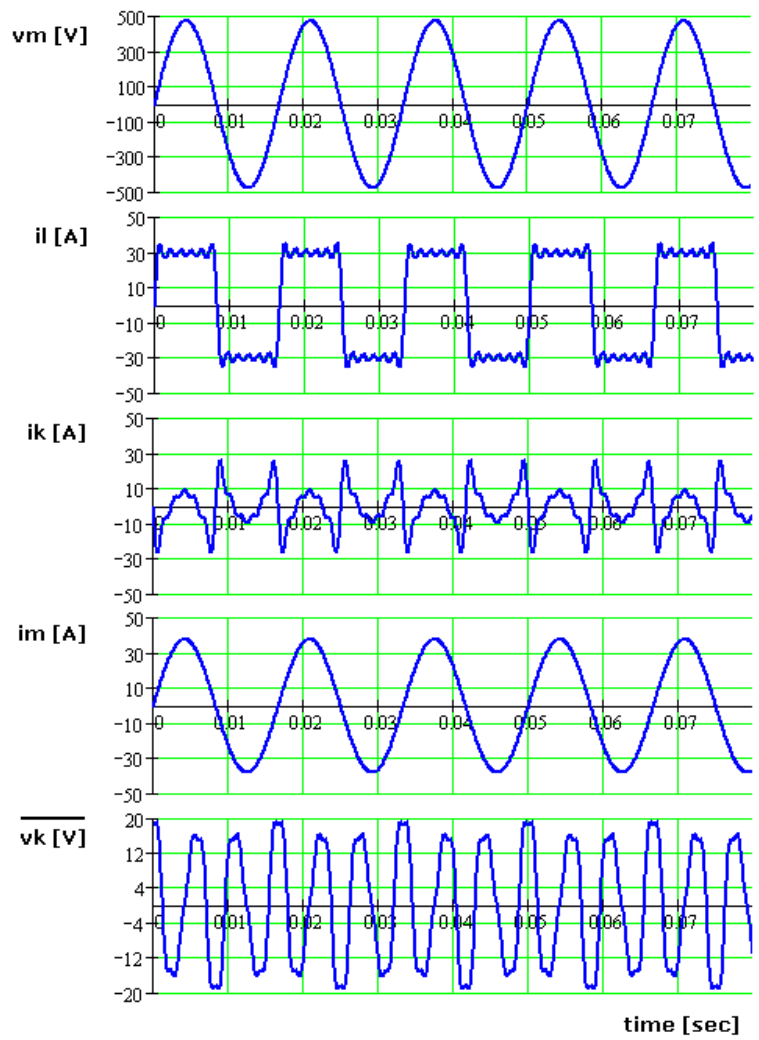

Fig. 9 - Simulation results, using the parameters displayed in Fig. 8 (mains voltage: 480V, single phase; load: $30 \mathrm{~A}$, square wave) 
This particular result is of extreme importance because it shows that is possible to design the filter for any nominal voltage adopted for the power converter, no matter how strict the load specifications are.

Some simulation results are presented, where it is possible to confirm the applicability of this solution, even in applications containing significant low order harmonic distortion.

The simulation results show that it is possible to operate the converter with peak to peak output voltage amplitude of $40 \mathrm{~V}$. This represents a 95\% reduction of the converter voltage, taking into consideration the mains voltage amplitude.

\section{Conclusion}

The introduction of an extra inductive element in the high-pass passive filter of an hybrid active shunt power filter topology conducts to slightly better results in what concerns to the reduction of the converter voltage.

This result permits the use of less expensive switching devices and energy storage components, and, or, the use of low voltage rated converters in medium voltage applications.

\section{References}

[1] Akagi, "Trends in Power Electronics and Motor Drives", in $5^{\text {th }}$ International Conference on Power Electronics and Drive Systems IEEE, 17-20 Nov. 2003, pp. 1- 7 Vol.1.

[2] L. Morán, J. Dixon, J. Espinoza \& R. Wallace "Using Active Power Filters to Improve Power Quality", 5th Brazilian Power Electronics Conference, COBEP'99, 1923 September 1999, pp 501-511.

[3] Ch.-Y. Hsu and H.-Y. Wu, "A new single-phase active power filter with reduced energy storage capacitor", Proceedings PESC'95, Jan 1996, pp. 202-208.

[4] Qiongq Chen, Z. Chen, Malcolm McCormick, "The application and optimization of C-type filter in a combined harmonic power filter”, Proceedings PESC'04, Aachen, Germany, 2004, pp.1041-1045. 